\title{
Dodecagonal quasicrystal in a polymeric alloy
}

$\operatorname{AUTHOR}(S)$ :

Dotera, T; Gemma, T

CITATION:

Dotera, T ...[et al]. Dodecagonal quasicrystal in a polymeric alloy.

Philosophical Magazine 2006, 86(6-8): 1085-1091

\section{ISSUE DATE:}

2006-02

URL:

http://hdl.handle.net/2433/50525

\section{RIGHT:}

This is not the published version. Please cite only the published version.; この論文は出版社版でありません。引用の際には出版社版を ご確認ご利用ください。 


\title{
Dodecagonal quasicrystal in a polymeric alloy
}

\author{
T. DOTERA† and T. GEMMA \\ $\dagger$ Kyoto University, Katsura, Kyoto 615-8510, Japan \\ †University of Tokyo, Komaba, Tokyo 153-8902, Japan
}

We report the formation of an approximant of a dodecagonal quasicrystal in a quasi-two-dimensional lattice Monte Carlo simulation of a star-shaped three component polymeric alloy. It is associated with the recent striking experimental manifestation of the complex Archimedean tiling $\left(3^{2} .4 .3 .4\right)$ consisting of triangles and squares, related to the $\sigma$ phase in the Frank-Kasper family, but whose edge length is about $80 \mathrm{~nm}$. The simulation box with periodic boundary conditions $(128 \times 128 \times 10)$ can be regarded as the Stampfli inflation of the $\left(3^{2} .4 .3 .4\right)$ tiling, an approximant of the dodecagonal quasicrystal. The corresponding edge length of deflated squares and triangles is thought to be about $300 \mathrm{~nm}$. Furthermore, the phason dynamics of the deflated square-triangle tiling is observed at an elevated temperature.

Keywords: quasicrystals; polymers; alloys; self-assembly; approximants; Monte-Carlo simulation

\section{Introduction}

Over the last twenty years, quasicrystals [1] with non-crystallographic (icosahedral [2], decagonal [3], octagonal [4], or dodecagonal [5,6]) rotational symmetry and quasiperiodic translational symmetry have been explored in the field of hard matter. Dodecagonal quasicrystals (DDQC) were found in small particles of $\mathrm{NiCr}$ [5] and in rapidly quenched NiV or NiVSi alloys [6]. These structures were explained by the tessellation of squares and equilateral triangles generated by an inflation method [7] or a higher-dimensional method [8]. Several simulations of DDQCs were performed for atomic systems and a square-triangle tiling system [9-12]. Tiling entropy associated with changing configurations of tiling was evaluated by a transfer matrix method [13] and by the Bethe anzatz [14]. Recently, the first stable DDQC of a layered structure of charcogenide TaTe was found [15] and analyzed by higher-dimensional crystallography [16]. Surprisingly, a 12-fold scattering pattern was reported for a liquid crystalline 
6PAGE

micellar phase of organic dendrons in the field of soft matter [17].

Meanwhile, in polymer sciences, microphase separations of 'block copolymers' composed of chemically distinct polymers linked together have provided magnificent crystalline morphologies such as lamellar, cocontinuous, cylindrical, and spherical phases. Furthermore, simulations [18-20] along with experiments [21] have demonstrated that $\mathrm{ABC}$ mikto-arm star-shaped block copolymers are very intriguing; their melt can be self-organized into fascinating two-dimensional tiling patterns. In addition, a recent remarkable experiment of an $\mathrm{ABC}$ star-shaped polymer alloy composed of polyisoprene, polystyrene and poly (2-vinylpyridine) revealed a complex Archimedean tiling phase denoted as $\left(3^{2} .4 .3 .4\right)$, or the $\sigma$ phase in the Frank Kasper family [22], composed of equilateral triangles and squares, whose edge length is about $80 \mathrm{~nm}$ [23]. Since the $\sigma$ phase is akin to DDQCs, DDQCs are expected to exist in polymeric systems. In this paper, we show that a dodecagonal quasicrystalline morphology appears in the Monte Carlo (MC) simulation of a lattice ABC star block copolymer system.

\section{Simulation method}

A simple extension of the bead-and-bond lattice polymer MC method called 'diagonal bond method [18]' is used. A bead occupies only one lattice point to ensure excluded volume interactions as usual. The difference is that the bond length can be $1, \sqrt{2}$ or $\sqrt{3}$ in the unit of lattice spacing. One ABC star block copolymer (figure 1) consists of $N_{\mathrm{A}}$ A-type beads, $N_{\mathrm{B}}$ B-type beads, $N_{\mathrm{C}}$ C-type beads, and one Y-type bead (junction point), which are connected by $N-1$ bonds, where $N=N_{\mathrm{A}}+N_{\mathrm{B}}+N_{\mathrm{C}}+1$. To represent energetics that drives the system to microphase separation, unit contact energies are imposed only between pairs of different species within the body diagonal distance $\sqrt{3}$ : We consider the Hamiltonian as $H=\Sigma \varepsilon_{i j}$, where $\varepsilon_{i j}=1$ when $i \neq j$, and $i$ and $j$ stand for A, B, C. The MC procedure is the following: We select one bead randomly and choose a trial move randomly out of possible moves; if the trial is a vacancy, we determine move or not according to the Metropolis algorithm.

Here we take $N_{\mathrm{A}}=9, N_{\mathrm{B}}=7$, and $N_{\mathrm{C}}=14$ or 16 . In order to see broad two-dimensional structures, whole simulations are carried out in a quasi-two-dimensional box with $L_{x}=L_{y}=128$ and $L_{z}=10$ subjected to periodic boundary conditions. The $\mathrm{C}$ component of a few star polymers can interact with themselves across 
6PAGE

$L_{z}$, leading to quick formation of cylinders parallel to the $z$-direction. The number of polymers in the system is $3724\left(N_{\mathrm{C}}=16\right)$ or $3964\left(N_{\mathrm{C}}=14\right)$, which is determined such that the occupation ratio of beads in the lattice points is 0.75 . The system is prepared as totally randomized at the infinite temperature, and then quenched at $\beta=1 / k_{\mathrm{B}} T=0.071$ to wait ordering, where $k_{\mathrm{B}}$ is the Boltzmann constant and $T$ is absolute temperature. This temperature was selected to retain ordering processes, or to get sufficient entropy.

\section{Simulation results}

In all simulations, cylindrical structures have developed. After a run of $10^{7}$ Monte Carlo steps (MCS) at $\beta=0.071$, we freeze structures at $\beta=0.2$ for $10^{5} \mathrm{MCS}$. In figure $2 \mathrm{a}$, the result for $\mathrm{A} 9 \mathrm{~B} 7 \mathrm{C} 14$ is shown. In the well-ordered region, we put vertices on the centre of $\mathrm{C}$ domains and draw lines, constructing the $\left(3^{2} .4 .3 .4\right)$ net composed of three triangles and two squares at every vertex. This crystalline structure is known as the $\sigma$ phase in the Frank-Kasper family, which has been found recently in a real polymeric system [23]. In figure $2 b$, we find local 12-fold-like wheel patterns, which remind us of DDQCs. Interestingly, they form a scaled $\left(3^{2} .4 .3 .4\right)$ lattice known as the Stampfli deflated lattice [7]. The periodic simulation box can be regarded as an 'approximant' [10] of the DDQC.

After the formation of the ordered structures, we have measured the structure functions of the C component, $\langle I(\boldsymbol{q})\rangle=\left\langle\left[\int \rho_{\mathrm{C}}(\boldsymbol{r}) e^{-i q \cdot r} \mathrm{~d} \boldsymbol{r}\right]^{2}\right\rangle$ averaged over $5 \times 10^{6}$ MCS at $\beta=0.071$. The density function $\rho_{\mathrm{C}}(\boldsymbol{r})$ is the averaged value over $10^{3} \mathrm{MCS}$ (see figure $3 \mathrm{a}$ ). The most prominent peaks (centre region) are located at $( \pm 2,0)$, $( \pm 2, \pm 1),( \pm 1, \pm 2),(0, \pm 2)$ in the unit of $2 \pi / A$, where $A$ is the lattice constant of the $\left(3^{2} .4 .3 .4\right)$ lattice [23]. On the other hand, the structure function shown in figure $3 \mathrm{~b}$ for A9B7C16 is evidently more circular and 12-fold, which is an evidence of the formation of the DDQC in a finite system. The same pattern has been obtained for A9B7C15 as well.

To quantify the order of 12-fold symmetry, we define a complex order parameter $\psi_{12}=\left[\Sigma_{q} e^{12 i \theta} I(q, \theta)\right] /\left[\Sigma_{q} I(q, \theta)\right]$, where $q$ and $\theta$ are the polar coordinates of $\boldsymbol{q}$; the sum is taken for the first shell of strong peaks $(q \leq 0.219 \pi)$, and only $I(q, \theta)>10$ is considered to eliminate backgrounds. The order parameter $\left|\psi_{12}\right|$ is plotted as a function of Monte Carlo steps in figure 3c. They grow and then stay almost constant after the formation of structures, which we have checked up to $2 \times 10^{7} \mathrm{MCS}$. We find 
6PAGE

that the parameter for A9B7C16 is slightly larger than that for A9B7C14 as expected.

In the usual square-triangle tiling, there is a sixfold node in the core of the wheel pattern. However, in figure $2 b$ there is no sixfold node. In the shell of the wheel patterns, fivefold starfish-like nodes are dominant, and we sometimes observe the starfish patterns even in the cores. From this, we deduce that the fivefold nodes are favorable locally in this system. However, as three regular pentagons cannot meet around a vertex without a tear, it is impossible to join three regular fivefold nodes together. To compromise, one remedy is to deform the fivefold node. In fact, a little flattened fivefold node is the elemental structure in $\left(3^{2} .4 .3 .4\right)$ (figure $\left.2 a\right)$. Another way is to introduce new degrees of freedom producing additional entropy. In the case of A9B7C16, we frequently find that neighboring $\mathrm{C}$ cells are dynamically connected and disconnected at $\beta=0.071$ (figure 4), meaning that the fivefold nodes and the wheel patterns are highly mobile. The wheel patterns change their positions. This collective dynamics can be viewed as a zipper rearrangement [10] of the square-triangle tiling as shown in figure 4. This dynamics in equilibrium is known as 'phason' dynamics, which is the crucial degree of freedom that quasicrystalline materials have [24-29]. It should be noted that even local rearrangement and deformation of mesoscopic domains occur, they keep the global symmetry and sharp diffraction peaks.

\section{Discussion}

Our numerical study suggests the possibility of polymeric DDQC formation. Clearly, the formation of a larger approximant is necessary to justify the present result. In addition, simulations at different temperatures are required to elucidate thermodynamic properties. For instance, to find whether the quasicrystal is a perfect [1] or random [10] tiling, the scaling analysis of phason fluctuations in three-dimensional simulations is necessary [26]. In particular, phason fluctuations along the cylindrical axis are of great interest, because they are indispensable for the random tiling approach [30].

To summarize, we have found a large-scale approximant of a dodecagonal quasicrystal in a quasi-two-dimensional Monte Carlo simulation of $\mathrm{ABC}$ star block copolymers. The scheme of square-triangle tilings is inapplicable at the lowest level of the microphase separation. At the Stampfli scaled level, however, the scheme is applicable. The edge length of a superimposed polygon is thought to be about $300 \mathrm{~nm}$, 
6PAGE

the region of photonic wavelengths, when the experimental value is assumed [23]. Furthermore, as a result of the collective motion of star polymers, the phason dynamics on a large scale can be constructed. Since the related crystalline phase has been found in an experimental system, it is quite natural to imagine that the realm of quasicrystals may extend to the polymeric alloy system.

\section{Acknowledgements}

We wish to thank Y. Matsushita and A. Takano for fruitful discussions. TD is indebted to the University of the Air for using computing facilities, where all simulations have been carried out.

\section{References}

[1] D. Levine and P. J. Steinhardt, Phys. Rev. Lett. 532477 (1984).

[2] D. Shechtman, I. Blech, D. Gratias, and J. W. Cahn, Phys. Rev. Lett. 531951 (1984).

[3] L. Bendersky, Phys. Rev. Lett. 551461 (1985).

[4] N. Wang, H. Chen, and K. H. Kuo, Phys. Rev. Lett. 591010 (1987).

[5] T. Ishimasa, H.-U. Nissen, and Y. Fukano, Phys. Rev. Lett. 55511 (1985).

[6] H. Chen, D. X. Li, and K. H. Kuo, Phys. Rev. Lett. 601645 (1988).

[7] P. Stampfli, Helv. Phys. Acta 591260 (1986).

[8] K. Niizeki and H. Mitani, J. Phys. A 20 L405-L410 (1987).

[9] P. W. Leung, C. L. Henley, and G. V. Chester, Phys. Rev. B 39446 (1989).

[10] M. Oxborrow and C. L. Henley, Phys. Rev. B 486966 (1993).

[11] M. Dzugutov, Phys. Rev. Lett. 702924 (1993).

[12] J. Roth, F.Gähler, Euro. Phys. J. B6 425 (1998).

[13] H. Kawamura, Physica A 17773 (1991).

[14] M. Widom, Phys. Rev. Lett. 70, 2094 (1993).

[15] M. Conrad, F. Krumeich, and B. Harbrecht, Angew. Chem. Int. Ed. 371384 (1998).

[16] A. Yamamoto, Acta Cryst. A 60142 (2004).

[17] X. Zeng, G. Ungar, Y. Liu, V. Percec, A. E. Dulcey and J. K. Hobbs, Nature 428 157 (2004).

[18] T. Dotera and A. Hatano, J. Chem. Phys. 1058413 (1996). 
6PAGE

[19] T. Gemma, A. Hatano, and T. Dotera, Macromolecules 353225 (2002).

[20] Y. Bohbot-Raviv and Z.-G. Wang, Phys. Rev. Lett. 853428 (2000).

[21] A. Takano, S. Wada, S. Sato, T. Araki, K. Hirahara, T. Kazama, S. Kawahara, Y. Isono, A. Ohno, N. Tanaka, and Y. Matsushita, Macromolecules 379941 (2004).

[22] F. C. Frank and J. S. Kasper, Acta. Cryst. 12483 (1959).

[23] A. Takano, W. Kawashima, A. Noro, Y. Isono, N. Tanaka, T. Dotera and Y. Matsushita, J. Polym. Sci. Part B: Polym. Phys. 432427 (2005).

[24] J. E. S. Socolar, T. C. Lubensky, and P. J. Steinhardt, Phys. Rev. B 34 3345(1986).

[25] Y. Ishii, Phys. Rev. B39 11862 (1989).

[26] T. Dotera and P. J. Steinhardt, Phys. Rev. Lett. 721670 (1994).

[27] K. Edagawa, K. Suzuki, and S. Takeuchi, Phys. Rev. Lett. 851674 (2000).

[28] G. Coddens, S. Lyonnard, and B. Hennion, Phys. Rev. B 626268 (2000).

[29] E. Abe, S. J. Pennycook, and A. P. Tsai, Nature 421347 (2003).

[30] H.-C. Jeong and P. J Steinhardt, Phys. Rev. B 489394 (1993).

\section{Figures}

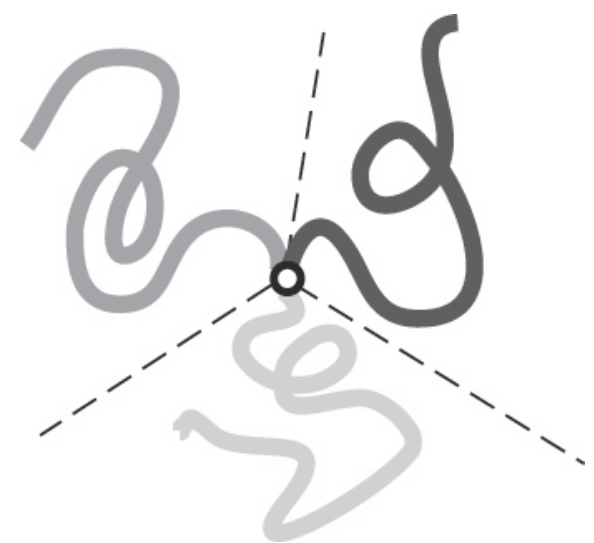

Figure 1. ABC star block copolymer consisting of a junction point and three arms of different chemical species A, B and C, which dislike each other. Between the three arms, three interfaces appear. 
6PAGE

(a)

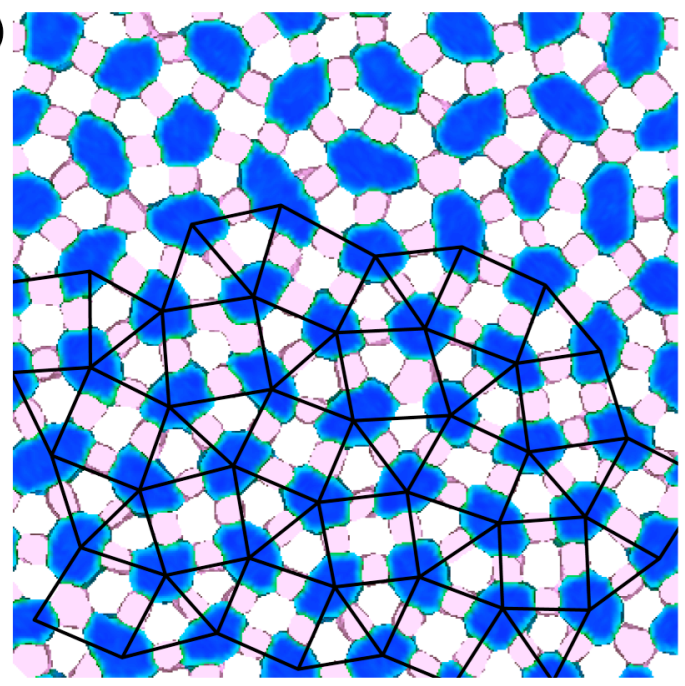

(b)

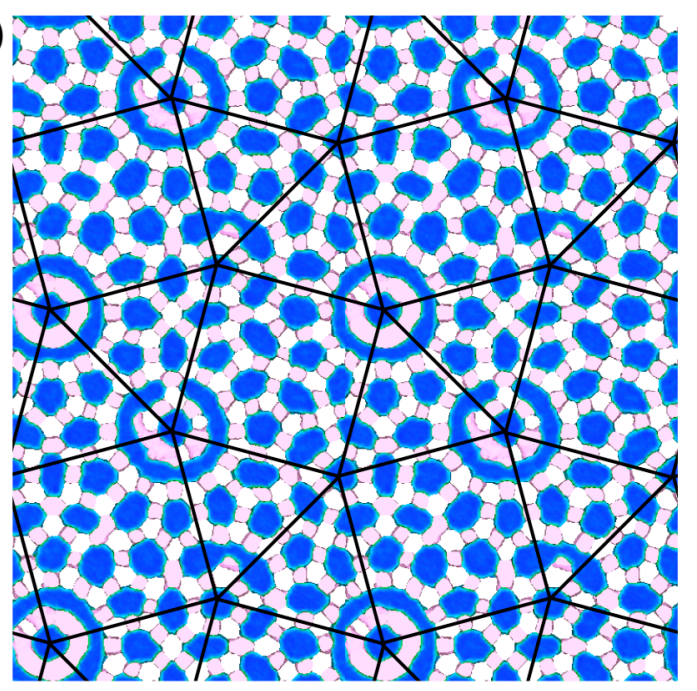

Figure 2. (a) Simulation result for A9B7C14 star block copolymers: A (transparent), B (light) and $\mathrm{C}$ (dark). A regular $\left(3^{2} .4 .3 .4\right)$ graph connecting centres of $\mathrm{C}$ cells is superimposed. Defects can be seen on the top. (b) Simulation result for A9B7C16 star block copolymers: A two-periodic unit cell made up of four replicas is rendered. Twelve-fold wheel patterns construct a magnified $\left(3^{2} .4 .3 .4\right)$ lattice (solid line), known as the Stampfli scaled lattice, which is regarded as a large approximant of the dodecagonal quasicrystal. Both pictures represent the volume rendering of averaged densities over $10^{3}$ MCS. 
6PAGE

(a)

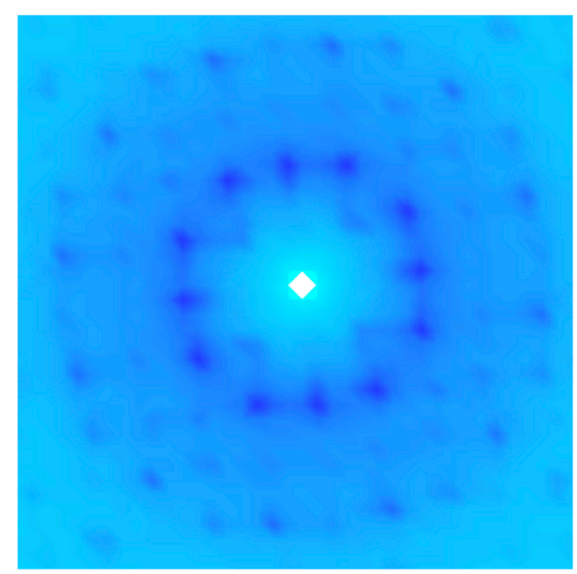

(b)

(c)



Figure 3. Structure functions for the C component $\log \langle I(\boldsymbol{q})\rangle$ for (a) A9B7C14 and (b) A9B7C16 star block copolymers. Almost 12-fold peaks are observed in (b). (c) Orientational order parameter $\left|\psi_{12}\right|$ as a function of Monte Carlo Steps (MCS). The dodecagonal case for A9B7C16 (open circles) is slightly larger than the $\left(3^{2} .4 .3 .4\right)$ case for $\mathrm{A} 9 \mathrm{~B} 7 \mathrm{C} 14$ (crosses). 
6PAGE

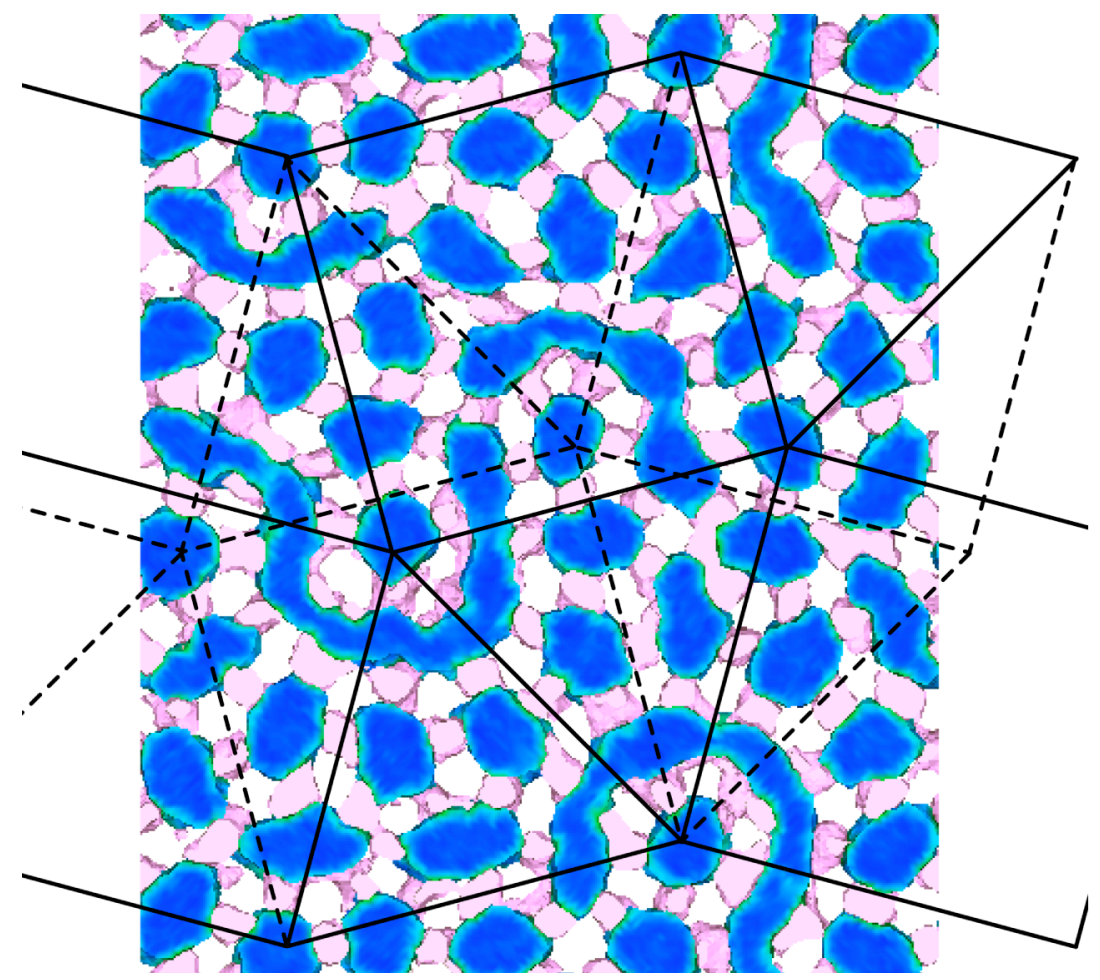

Figure 4. Dynamic phasons in the A9B7C16 system. Superimposed lines represent tiling rearrangement from broken lines to solid lines. 